\title{
The Formation of Jupiter's Diluted Core by a Giant Impact
}

Shang-Fei Liu ${ }^{1,2 \star}$, Yasunori Hori ${ }^{3,4}$, Simon Müller ${ }^{5}$, Xiaochen Zheng ${ }^{6}$, Ravit Helled ${ }^{5}$, Doug Lin ${ }^{7,8} \&$ Andrea Isella ${ }^{2}$

${ }^{1}$ School of Physics and Astronomy, Sun Yat-sen University Zhuhai Campus, 2 Daxue Road, Tangjia, Zhuhai 519082, Guangdong Province, P.R. China;

${ }^{2}$ Department of Physics and Astronomy, Rice University, 6100 Main St., MS-108, Houston, TX 77005, USA;

${ }^{3}$ Astrobiology Center, 2-21-1 Osawa, Mitaka, Tokyo 181-8588, Japan;

${ }^{4}$ National Astronomical Observatory of Japan, 2-21-1 Osawa, Mitaka, Tokyo 181-8588, Japan;

${ }^{5}$ Institute for Computational Science, Center for Theoretical Astrophysics and Cosmology, University of Zurich, Winterthurerstrasse 190, CH-8057 Zurich, Switzerland;

${ }^{6}$ Department of Physics and Center for Astrophysics, Tsinghua University, Beijing 10084, P.R. China;

${ }^{7}$ Department of Astronomy and Astrophysics, University of California, Santa Cruz, CA 95060, USA;

${ }^{8}$ Institute for Advanced Study, Tsinghua University, Beijing 100084, P.R. China.

${ }^{\star}$ To whom correspondence should be addressed; Email: liushangfei@mail.sysu.edu.cn

The Juno mission ${ }^{1}$ is designed to measure Jupiter's gravitational field with an extraordinary

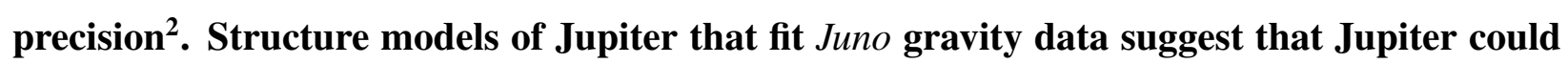
have a diluted core and a total heavy-element mass $M_{\mathrm{Z}}$ ranging from ten to two dozens of Earth masses $\left(\sim 10-24 M_{\oplus}\right)$. In that case the heavy elements are distributed within an extended region with a size of nearly half of Jupiter's radius $R_{\mathfrak{j}} \sqrt{34}$. Planet formation models indicate that most of the heavy elements are accreted onto a compact core ${ }^{5+7}$, and that almost no solids are accreted during runaway gas accretion (mainly hydrogen and helium, hereafter H-He), regardless to whether the accreted solids are planetesimals or pebbles $\sqrt{8}-10$. Therefore, the inferred heavy-element mass in the planet cannot significantly exceeds the core's mass. The fact that Jupiter's core could be diluted, and yet, the estimated total heavy-element mass in the planet is relatively large challenges planet formation theory. A possible explanation is erosion of the compact heavy-element core. Its efficiency, however, is uncertain and depends on both the immiscibility of heavy materials in metallic hydrogen and the efficiency 
of convective mixing as the planet evolves ${ }^{11,12}$. Neither can planetesimal enrichment and vaporization ${ }^{13-15}$ produce such a large diluted core. Here we show that sufficiently energetic head-on collisions between additional planetary embryos and the newly emerged Jupiter can shatter its primordial compact core and mix the heavy elements with the outer envelope. This leads to an internal structure consistent with the diluted core scenario which is also found to persist over billions of years. A similar event may have also occurred for Saturn. We suggest that different mass, speed and impact angle of the intruding embryo may have contributed to the structural dichotomy between Jupiter and Saturn ${ }^{16}-18$.

Giant impacts $\frac{1920}{20}$ are likely to occur shortly after runaway gas accretion when a gas giant planet's gravitational perturbation significantly intensifies (about thirty-time increases in a fraction of a million years) and therefore destabilizes the orbits of nearby protoplanetary embryos. This transition follows oligarchic growth ${ }^{21}$ and the emergence of multiple embryos with isolation mass in excess of a few $M_{\oplus}{ }^{22}$. Some of these massive embryos may collide with the gas giant during their orbit crossing $23 \mid 24$. Through tens of thousands of gravitational $N$-body simulations with different initial conditions such as Jupiter's growth model, orbital configuration, etc. (see Methods), we find that an emerging Jupiter has a strong influence on nearby planetary embryos. As a result, a significant fraction of these embryos could collide with Jupiter within a few million years, i.e., within the Solar nebula lifetime. Among those catastrophic events, head-on collisions are more common than grazing ones due to Jupiter's gravitational focusing effects.

In order to investigate the influence of such impacts on the internal structure of the young Jupiter we use the hydrodynamics code $\mathrm{FLASH}^{25}$ with the relevant equation of state (EOS). Details of the computational setup and the simulations are presented in the Methods section. Generally, the disintegration of the intruding embryo leads to the disruption of the planet's original core. However, to establish a large diluted-core structure as inferred from recent Jupiter structure models based on Juno's measurements, the core and embryos' fragments need to efficiently mix with the surrounding convective envelope, which requires a large embryo to strike the young Jupiter almost head-on. Massive embryos are available at the advanced stage of Jupiter's formation and our $N$ body simulations also suggest that head-on collisions are common (see Methods).

In Figure 1 we show the consequence of a head-on collision between an embryo and Jupiter with an initial $M_{\text {core }}=10 M_{\oplus}$ silicate/ice core, a H-He envelope, approximately present-day total 
mass and radius (The young Jupiter may have a size up to twice of its current-day value, however, to avoid introducing additional free parameters, we consider models more similar to current Jupiter). In fact, the post-impact core-envelope structure mainly depends on the mass of the initial core and envelope as well as the impactor's mass and impact velocity $V_{\mathrm{imp}}$. We adopt an impact speed $V_{\text {imp }} \sim 46 \mathrm{~km} \mathrm{~s}^{-1}$ which is close to the free-fall speed onto Jupiter's surface (see Methods) and assume the impactor is comprised of an $8 M_{\oplus}$ silicate-ice core and a $2 M_{\oplus} \mathrm{H}$-He envelope. The total mass of proto-Jupiter's and embryo's core $M_{\mathrm{Z} \text {,total }}$ is chosen to be compatible with the derived mass of heavy elements in Jupiter's diluted core models 3 . Note that at Jupiter's distance of 5.2 AU from the sun, the impactor's speed relative to the gas giants is limited by the planets' surface escape speed. The acquisition of protoplanetary embryos would not lead to any major changes in the spin angular momentum and orientation of the targeted planet. The total energy injected into the young Jupiter by the intruding embryo is only a few percent of its original values so that there is little change in Jupiter's mean density and mass.

The impact results in little mass loss (see Table 1), while Jupiter's initial core is completely disrupted. During the impactor's plunge and collision with the primordial core, a large amount of kinetic energy is dissipated. The heat release near the center increases the local temperature $T$, offsets the pressure $P$ balance, and induces oscillations (see the full video in Supplementary Information). The steep negative entropy gradient near the core overturns the local negative molecular weight gradient $\mu$ and leads to convection in the inner part of the envelope. Vigorous turbulence stirs up efficient mixing between the heavy elements and $\mathrm{H}-\mathrm{He}$ envelope. After a few dynamical time-scales (a characteristic time scale to measure expansion or contraction of a planet; Jupiter's dynamical time scale is roughly a third of an hour), the initial silicate/ice core is thoroughly homogenized with the surrounding $\mathrm{H}-\mathrm{He}$ and their mass fraction $Z \leq 0.5$ interior to $\sim 0.2 R_{\mathrm{J}}$. Within $\sim 30$ dynamical time-scales, Jupiter's interior settles into a quasi-hydrodynamic equilibrium with a diluted core extending to $R \sim 0.4-0.5 R_{\mathrm{J}}$ (see Table 1 and panel a of Figure 2 ). In the outer half of the envelope, the gas density is slightly elevated and a small trace of the dredge-up heavy elements $(Z)$ leads to the formation of a composition gradient.

The post-impact heavy-element distribution leads to a composition gradient that could evolve and become similar to an internal structure with a diluted-core. However, the hydrodynamic simulation is terminated ten hours after the impact. In order to explore under what conditions a dilutedcore-like structure persists after the 4.56 Gyrs of Jupiter's evolution, we compute the thermal- 
evolution shortly after the impact until today. The hydro-simulation sets the initial heavy-element gradient as shown in panel a of Figure 2. Since the post-impact temperature profile is unknown*? we consider various temperature profiles with different central temperatures. Furthermore, we consider an initial thermal structure that accounts for the accretion shock during runaway gas accretion as suggested by a recent Jupiter formation model ${ }^{27}$ (see Methods for details). We find that for the head-on collision, a post-impact central temperature of $\sim 30,000 \mathrm{~K}$ leads to a current-state Jupiter with a diluted core. Another pathway to Jupiter's diluted core is if the initial temperature profile is shaped by the accretion shock. In panel b of Figure 2 we show the density profiles of the best-fitting models after the 4.56 Gyrs of evolution. If the central temperatures are higher (e.g., 50,000K), the interior is hot enough to "delete" the heavy-element gradient leading to a fully mixed planet. On the other hand, for low central temperatures $(\sim 20,000 \mathrm{~K})$, convective mixing is less significant and the inferred density profile is less consistent with a diluted-core structure. Therefore, we conclude that Jupiter's diluted-core structure could be explained by a giant impact event, but under some specific conditions which include a head-on collision with a massive planetary embryo, a postimpact central temperature of $\sim 30,000 \mathrm{~K}$, or an initial thermal structure created by the accretion shock during the runaway phase. Indeed, the hydrodynamic simulation implies that most of the impact energy is not deposited in the deep interior which results in lower central temperatures and to a diluted-core solution (see Methods).

In contrast, if the same embryo collides with Jupiter at a grazing angle, it would be tidally disrupted gradually while sinking towards the center (see Figure 3). In Methods, we further show that impactors with Earth or sub-Earth mass disintegrate in gas giants' envelope before reaching their centers. Without smashing into the core directly, the shock wave induced by the impactor alone is insufficient to homogenize Jupiter's interior. These impacts generally lead to core growth rather than core destruction. Since impacts of planetary embryos are expected to be common after a gas giant's runaway gas accretion phase, a similar event with different impact conditions may have also happened to Saturn, and could in principle explain the dichotomy between the internal structures of Jupiter and Saturn $16-19$. A gradual accretion of planetesimals along with the runaway gas accretion may also produce a diluted core ${ }^{15,28}$. A relevant issue to be investigate elsewhere is whether the steep compositional gradient needed to preserve the diluted core can also be established after a series of planetesimal-accretion events rather than a single embryo's giant impact. Finally, extra-solar gas giant planets could also suffer giant impacts which could explain

\footnotetext{
*the exact temperature profile depends on the formation process 2627 , the energetics of the impact, etc.
} 
some of the giant exoplanets with extremely large bulk metallicities ${ }^{29}$.

Acknowledgements We thank S.M. Wahl and Y. Miguel for sharing their results with us. We thank J.J. Fortney, P. Garaud and H. Rein for helpful conversations. S.-F.L. thanks the support and hospitality from Aspen Center for Physics during the early stage of this work. D.L. thanks Institute for Advanced Study, Princeton, Institute of Astronomy and DAMTP Cambridge University for support and hospitality when this work was being completed. R.H. acknowledges support from SNSF grant 200021_169054. A.I. acknowledges support from the National Aeronautics and Space Administration under award No. 80NSSC18K0828 and from the National Science Foundation under grant No. AST-1715719.

Author Contributions D.L. had the impact idea. S.-F.L. and A.I. examined its feasibility. S.F.L. coordinated this study. S.-F.L. and Y.H. designed and analysed the hydrodynamic simulations. X.Z. and S.-F.L. performed and analysed the $N$-body simulations. S.M. and R.H. designed the long-term thermal evolution study. All authors contributed to discussions, as well as editing and revising the manuscript.

Author Information The authors declare that they have no competing financial interests. Correspondence and requests for materials should be addressed to S.-F.L. (email: liushangfei@ mail.sysu.edu.cn). 
a Befrore impact

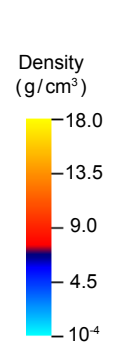

b During impact

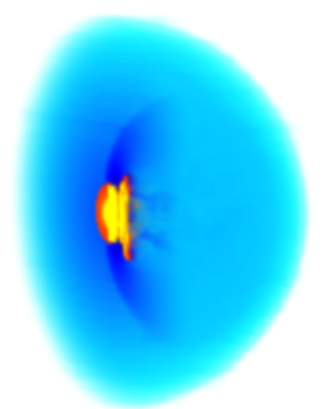

C After impact

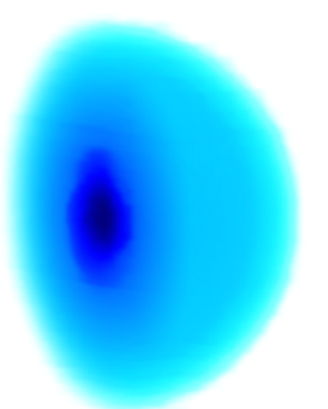

Figure 1: 3D cutaway snapshots of density distributions during a merger event between a proto-Jupiter with a $10 M_{\oplus}$ rock/ice core and a $10 M_{\oplus}$ impactor. a, just before the contact. b, the moment of core-impactor contact. c, 10 hours after the merger. Due to impact-induced turbulent mixing, density of Jupiter's core decreases by a factor of three after the merger, resulting in an extended diluted core. A 2D presentation of density slices of the same event is shown in Extended Data Figure 3. 

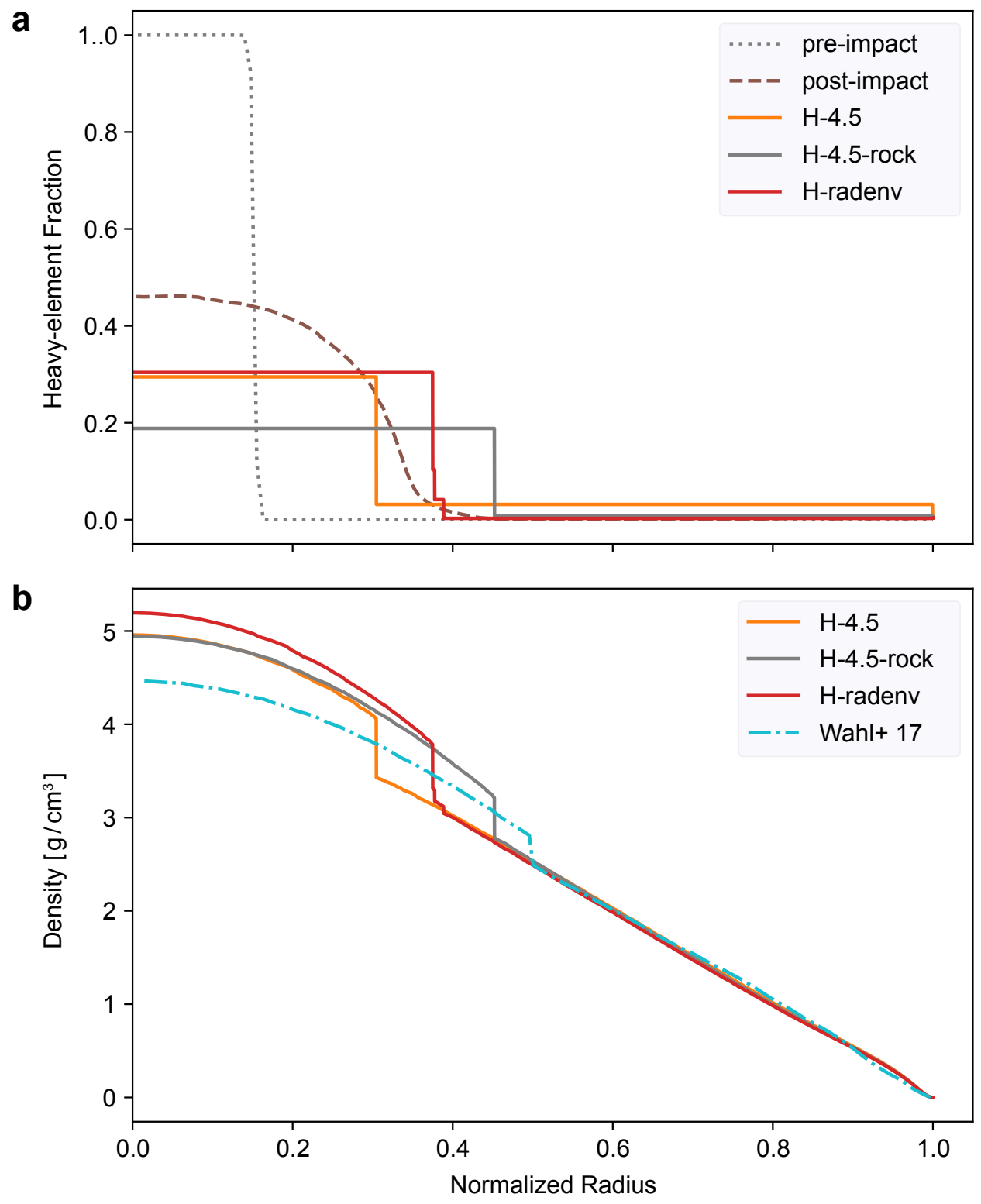

Figure 2: Post-impact thermal evolution models. a, Heavy-element distribution vs. normalized radius before (dotted) and after (dashed) the giant impact. The solid lines show the composition after 4.56 Gyrs of evolution for the three best-fit models that result in a diluted core, see the Methods section and Table 3 for more details. b, Density vs. normalized radius after 4.56 Gyrs of evolution (solid) and from the diluted-core interior structure model of Wahl et al. 2017 ${ }^{3}$ (dashdotted). 
Table 1: Initial conditions and final outcomes of the head-on giant impact simulation. $M_{\mathrm{T}}$ and $M_{\mathrm{I}}$ are the total mass of the proto-Jupiter and the impactor, respectively. $M_{\text {core }}$ is the mass of heavy elements in the proto-Jupiter's core. $M_{Z, \mathrm{I}}$ and $M_{Z \text {,total }}$ are the total mass of heavy elements contained in the impactor and the system, respectively. After the merger, values of total mass of Jupiter $M_{\mathrm{T}}$ and heavy elements $M_{Z \text {,total }}$ are measured within 1 and $2 R_{\mathrm{J}}$, respectively. Those values reveal that the majority of Jupiter's mass still resides in its original size, albeit a hot extended lowdensity envelope mostly made of H-He forms after the merger (see also Extended Data Figure 3). The size of a diluted core was defined as the central region enclosed by a sphere with $Z>0.014$. The last three rows list values for the best-fit evolution models to the interior structure model of Jupiter with a diluted core ${ }^{3}$. $R_{\text {core }} / R_{\mathrm{J}}$ is the radius of the proto-Jupiter's core scaled to the Jupiter's current radius. All mass quantities are in unit of $M_{\oplus}$.

\begin{tabular}{c|cccccc}
\hline & $M_{\mathrm{T}}$ & $M_{\text {core }}$ & $M_{\mathrm{I}}$ & $M_{Z, \mathrm{I}}$ & $M_{Z, \text { total }}$ & $R_{\text {core }} / R_{\mathrm{J}}$ \\
\hline \hline Before merger & 306.714 & 9.962 & 9.967 & 7.975 & 17.937 & 0.15 \\
$\sim 10$ hrs after merger & $304.946 / 313.360$ & 17.693 & - & - & $17.901 / 17.925$ & 0.423 \\
H-4.5: After 4.56 Gyrs & 313.36 & 10.61 & - & - & 17.925 & 0.30 \\
H-radenv: After 4.56 Gyrs & 313.36 & 17.24 & - & - & 17.925 & 0.39 \\
H-4.5-rock: After 4.56 Gyrs & 313.36 & 15.92 & - & - & 17.925 & 0.45 \\
\hline
\end{tabular}




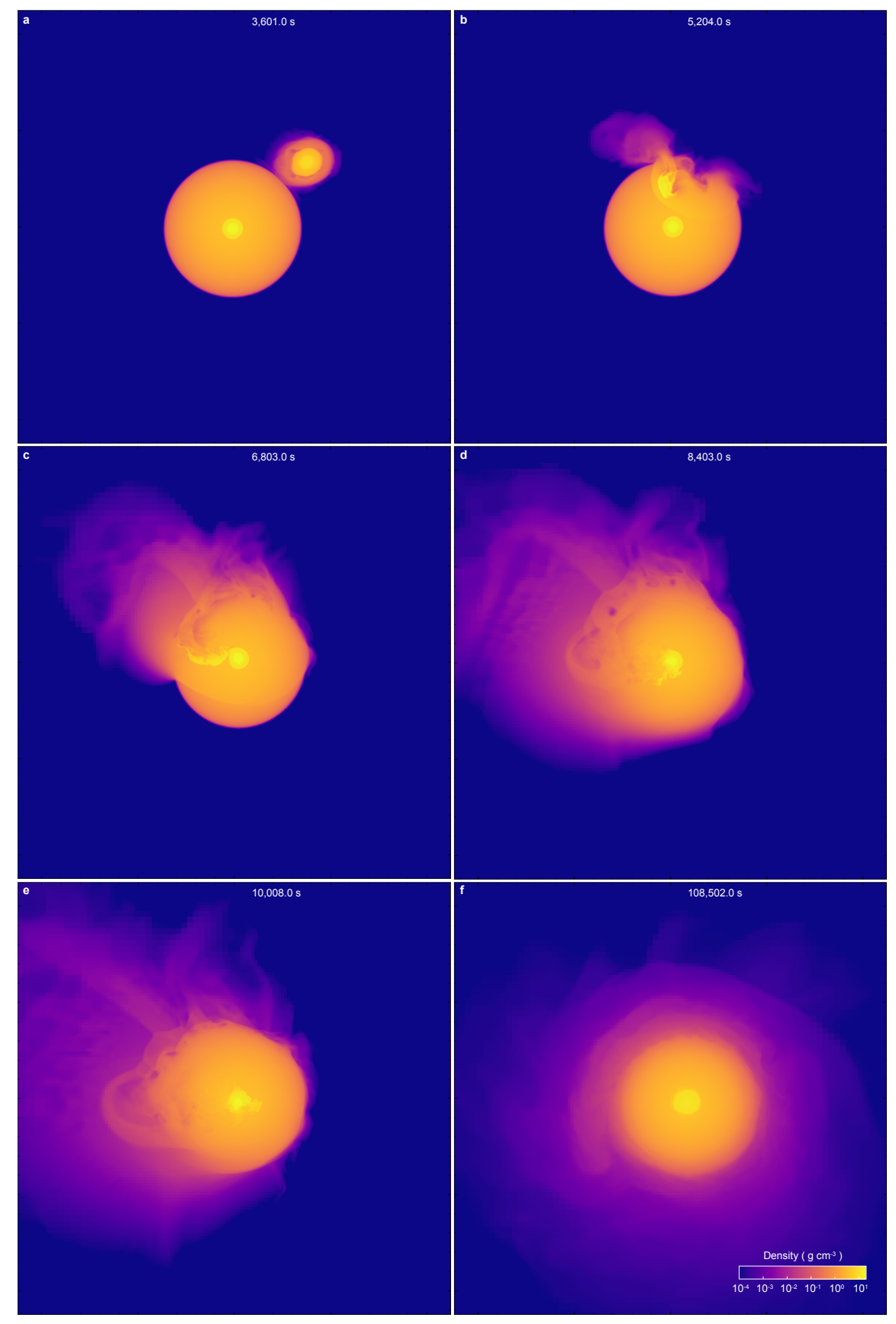

Figure 3: 2D snapshots of an off-center collision between the proto-Jupiter with a $10 M_{\oplus}$ solid core and a $10 M_{\oplus}$ impactor. a, Density contours in the orbital plane before the impact; b-e, the impactor being disrupted and accreted; e, at $\sim 30$ hours after the impact. See Methods for detailed discussion. 


\section{Methods}

A statistical $N$-body study of embryo collisions: We investigate the statistics of collisions between an emerging Jupiter and planetary embryos with the open-source $N$-body code REBOUND ${ }^{30}$ version 3.6.2. To simulate the evolution of a planetary system we choose the built-in hybrid HERMES integrator ${ }^{\dagger}$, which uses the WHFast integrator ${ }^{\sqrt{31}}$ for the long-term dynamics and switches to the IAS15 integrator ${ }^{\frac{32}{12}}$ when close encounters (such as scattering and collisions) happen.

Our $N$-body simulations start from a coplanar configuration in which five ten-Earth-mass planetary embryos $\left(M_{\mathrm{p}}=10 M_{\oplus}\right)$ orbit the $\operatorname{Sun}\left(M_{*}=1 M_{\odot} \sim 3.3 \times 10^{5} M_{\oplus}\right)$ on circular prograde orbits. The embryo at 5.2 astronomical units (a.u.) from the Sun grows into a Jupiter-mass planet at the end of the simulation. Initially, two embryos are placed interior to Jupiter's orbit and the other two embryos are placed exterior to Jupiter's orbit. The orbital separation between two adjacent embryos $i$ and $i+1$ is determined by a dimensionless number

$$
k=\frac{2 a_{i}}{r_{\mathrm{H}}}\left(\frac{a_{i+1}-a_{i}}{a_{i+1}+a_{i}}\right),
$$

where $a_{i}$ and $a_{i+1}$ are the semi-major axes of each embryo, and $r_{\mathrm{H}}=a_{i}\left(\frac{M_{\mathrm{p}}}{3 M_{*}}\right)^{1 / 3}$ is the Hill radius of embryo $i$. It is convenient to express Equation 1 in terms of $q=a_{i+1} / a_{i}$, the ratio of semi-major axis between embryos $i$ and $i+1$

$$
k=2\left(\frac{3}{\mu}\right)^{1 / 3}\left(\frac{q-1}{q+1}\right),
$$

where $\mu=M_{\mathrm{p}} / M_{*} \simeq 3 \times 10^{-5}$ is the mass ratio between the embryo and the Sun. A larger $k$ will give rise to a wider separation, i.e. a more dynamically stable configuration. Extended Data Table 1 summarizes the locations of all embryos for a given parameter $k$ in our $N$-body simulation suite. In addition, we also consider a configuration, in which all four embryos are beyond Jupiter's orbit.

At the onset of the simulation, the runaway gas accretion of Jupiter's core starts. The mass accretion rate is an exponential decay function characterized by an exponential time parameter $t_{\text {grow }}$ ranging from $0.1 \mathrm{Myr}$ to $0.5 \mathrm{Myr}$ in this study. At a given time $t$, the mass of an emerging Jupiter is determined by

$$
M(t)=M_{\mathbf{J}}-\left(M_{\mathrm{J}}-M_{\mathrm{p}}\right) e^{-t / t_{\text {grow }}}
$$

\footnotetext{
${ }^{\dagger}$ In recent updates of REBOUND, the HERMES integrator has been replaced by the MERCURIUS integrator, which offers a similar capability in a single scheme.
} 
where $M_{\mathrm{J}}=317.8 M_{\oplus}$ is one Jovian mass. In this model, Jupiter quickly acquires more than $90 \%$ of its mass within $3 t_{\text {grow }}$ and steadily gains another a few percent of its mass until $t=10 t_{\text {grow }}$. For simplicity, we assume that all other four embryos do not grow during the whole time, since a typical hydrostatic growth stage of an embryo before it entering the runaway gas accretion phase is around a few Myr and the embryo mass barely increases.

Size is another crucial factor as a larger cross-section can boost the probability of collisions. We adopt the mean density of the Earth for embryos, so their sizes $R_{\mathrm{p}} \simeq 2.15 R_{\oplus}$, where $R_{\oplus}$ is Earth's mean radius. For the emerging Jupiter, its mean density could be as low as half of its current-day value. We use the parameter $f$ to describe the degree of inflation.

Thus, we design a simple classification for our $N$-body simulation suite with three free parameters $k, t_{\text {grow }}$ and $f$. For each combination set of $\left(k, t_{\text {grow }}, f\right)$, we run thousands of simulations with other orbital parameters (e.g., true anomaly, argument of periapsis) randomly chosen between 0 and $2 \pi$.

At the end of an $N$-body simulation ( $t=10 t_{\text {grow }}$ ), a planetary embryo may remain bound to the Sun with considerable changes in its orbit, or coalesce with Jupiter and other embryos or escape from the system after a close encounter. The statistics of final outcomes of four planetary embryos under the influence of an emerging Jupiter is shown in Extended Data Figure 1. The results are grouped by different parameters to compare their impacts. In all subsets of our $N$ body simulations, we observe an efficient pathway to deliver planetary embryos to collide with an emerging Jupiter.

Because embryos are equally distributed on both sides of Jupiter's orbit (except for the last group with all embryos in the "Outward" state to begin with), the results suggest that embryos both interior or exterior to Jupiter could collide with Jupiter within the simulation time. While embryos beyond Jupiter may have a slightly larger chance to strike Jupiter as there are less embryos asymptotically result in an "Outward" destiny. Among the three key parameters, orbital tightness characterized by $k$ plays the most substantial role in affecting the collision probability. For the same orbital configuration, Jupiter inflation factor $f$ can slightly change the collision rate. However, Jupiter's accretion history determined by $t_{\text {grow }}$ has the least influence on the results. 
We analyze the distribution of collision angle using our $N$-body simulation suite. And the histograms of collision angles are plotted in Extended Data Figure 2. Each histogram represents a detailed breakdown of "Merger" events of a simulation set presented in Extended Data Figure 1. Unlike collisions between similar-sized planetary bodies, in which $45^{\circ}$ collisions are common ${ }^{33}$, the statistical results suggest that half of the merger events have collision angles less than $\sim 30^{\circ}$ in all cases we investigated. We suggest that low-angle impacts are very common because of Jupiter's strong gravitational focusing effect.

It is often useful to define a two-body escape velocity as

$$
V_{\mathrm{esc}}=\left(\frac{2 \mathrm{G}\left(M_{\mathrm{J}}+M_{\mathrm{p}}\right)}{R_{\mathrm{R}}+R_{\mathrm{p}}}\right)^{1 / 2},
$$

which is around $51 \mathrm{~km} \mathrm{~s}^{-1}$ for the proto-Jupiter and the $10 M_{\oplus}$ impactor studied in the hydrodynamic simulation. In general, an embryo's impact velocity $V_{\text {imp }}$ is related to $V_{\text {esc }}$ as well as the local Keplerian velocity $V_{\text {kep }}$. Gravitational perturbation during close encounters can produce an impact velocity with a magnitude up to the escape velocity 34 . On the other hand, the Keplerian orbital velocity gives rise to the random velocity dispersion during impacts. At Jupiter's current location, $V_{\text {kep }} \sim 13 \mathrm{~km} \mathrm{~s}^{-1}$ is much smaller than $V_{\text {esc }}$, so the impact velocity $V_{\text {imp }}$ is approximately at the escape velocity $V_{\text {esc }}$. Indeed, we find the impact velocity is quantitatively similar to $V_{\text {esc }}$ rather than $V_{\text {kep }}$, although $V_{\text {imp }}$ is always slightly smaller than $V_{\text {esc }}$ in the $N$-body simulation suite, because initial separations between Jupiter and embryos are finite (a two-body system has a negative gravitational potential energy).

This simple statistical model can be improved in the future to compare with other formation models of the outer Solar system. For example, because Jupiter's inward migration is much slower than those planetary embryos, the presence of Jupiter in the Solar nebula acts like a barrier for inward migrating planetary embryos formed exterior to Jupiter ${ }^{35}$. Consequently, collisions among those planetary embryos may become frequent and some of those events may eventually form Uranus and Neptune $e^{36}$.

Hydrodynamic simulations: Our 3D hydrodynamic simulation of giant impacts between a protoJupiter and a protoplanetary embryo is based on the framework of the Eulerian FLASH code ${ }^{25}$ which utilizes the adaptive-mesh refinement. The setup of giant impact simulations has been laid out in our previous study 37 . Here, we briefly describe the model of the planetary interior. The 
primordial Jupiter is modeled with a three-layer structure: a silicate core, an icy mantle, and a H-He envelope. We calculate two thermodynamic (density and internal energy) properties of silicate and ice material and their velocities with the governing continuity, momentum, and energy equation. For computational efficiency, these quantities are converted into pressure and temperature with the Tillotson $\operatorname{EOS}^{38}$. The mass fraction between ice to silicate is assumed to be 2.7 according to that of protosun (2-3). In addition, the $\mathrm{H}-\mathrm{He}$ EOS is modeled with an $n=1, \gamma=2$ polytropic relation, where $n$ and $\gamma$ are the polytropic and adiabatic indexes. Although this idealized treatment ignores effects such as the $\mathrm{H}-\mathrm{He}$ phase transition and separation, it reasonably matches the density profile of Jupiter's envelope calculated with ab-initio $\operatorname{EOS}^{39}$, and is good enough for dynamic processes that happen in a few hours (see detailed discussion below).

Collisions between a proto-Jupiter with a $10 M_{\oplus}$ core and a $10 M_{\oplus}$ embryo: From $N$-body simulations we learn that most collisions have collision angles less than 30 degrees, so we first study the head-on collision as one of the representative cases in the main text and the consequence is shown in Figure 1. Here we also plot its 2D counterpart in Extended Data Figure 3. The general behavior of head-on collisions has been studied extensively in previous works 20,37 . To recapitulate, the solid material of the impactor can penetrate Jupiter's gaseous envelope and smash into its core as a whole. As a result, Jupiter's core gets completely destroyed after the impact. The release of a large amount energy inside the proto-Jupiter drives large scale turbulence and the primordial compact core is homogenized subsequently. We compare the enclosed internal energy of Jupiter as a function of radius before and after the impact. The results are shown in Extended Data Figure 4. Although Jupiter gains internal energy through the release of kinetic and gravitational energy of the impactor as well as impactor's own internal energy, the core region gets barely heated. In fact, there is even a little decrease of internal energy inside the core region right after the impact possibly due to mixing with $\mathrm{H}-\mathrm{He}$. The analysis suggests that the impactor dumps most of its energy outside the original core region.

Our simplified EoS for $\mathrm{H} / \mathrm{He}$ causes less efficient dissipation of the impactors kinetic energy within the $\mathrm{H} / \mathrm{He}$ envelope. As a vigorous mixing between $\mathrm{H} / \mathrm{He}$ and core material, however, is driven by a merger between the core of a photo-Jupiter and an impactor, we can expect formation of a dilute core to occur regardless of EoS models. In addition, a temperature profile inside a core is not strongly affected by the choice of a H/He EoS model because the impact causes only a small change in internal energy inside the core. 
To illustrate the effects of off-center collisions, we run the same setup of simulation except that the collision angle is at 45 degrees. The consequence is shown in Figure 3. Because the initial impact velocity is at the escape velocity, the impactor misses Jupiter's core and overshoots until Jupiter's gravitational force pulls it back. During its course, the impactor gradually loses angular momentum and gets torn apart. The remnant is gently accreted by Jupiter's solid core later on. As a result, the impact has little influence on Jupiter's core-envelope structure.

A head-on collision between a proto-Jupiter with a massive core and a small impactor: In addition, we perform a head-on collision between a proto-Jupiter with a massive primordial core of $17 M_{\oplus}$ and a $1 M_{\oplus}$ impactor, which is composed of pure silicate, at the same impact velocity. The total amount of heavy elements is the same as that in previous head-on and off-center models (hereafter, case-1 and case-2). Unlike case-1, the impactor disintegrates in the proto-Jupiter's envelope before making contact with the core. A strong shock wave induced by the entry of the impactor propagates throughout the entire planet and deforms the core (see panel c of Extended Data Figure 5). The heavy elements are well mixed with a small fraction of $\mathrm{H}-\mathrm{He}$ (only $\sim 5 \mathrm{wt} \%$ ) inside the proto-Jupiter's core after the impact because of a weak impact-induced oscillation and less efficient turbulent mixing. As a result, the central density of the core still decreases by a factor of two thirds. Although the core-envelope boundary slightly spreads out, a steep density gradient between the core and $\mathrm{H}-\mathrm{He}$ envelope is preserved, leading to the retention of a compact, massive core.

To summarize, only in case-1 we observe a smooth transition between the core and H-He envelope after the impact, as the impactor is massive and hits Jupiter's core directly. However, in both case- 2 and case- 3 , because the impactor is unable to collide with the core as an integrated body, the proto-Jupiter's core becomes less chemically homogenized after it gets restored from deformation. Therefore, we conclude that neither a small impactor, nor an off-center collision is able to form a large diluted core, and proto-Jupiter with a primordial solid core should have experienced a catastrophic nearly head-on collision with a large embryo, if the present-day Jupiter has a massive, diluted core. A more comprehensive parameter study, including a range of impactor's mass and speed as well as off-center collisions, will be presented elsewhere.

Post-impact thermal evolution: We simulate Jupiter's long-term evolution after the giant impact in order to identify the evolutionary paths that lead to a diluted core structure at present-day. The 
planetary evolution is modelled using the 1D stellar evolution code Modules for Experiments in Stellar Astrophysics (MESA), where the planet is assumed to be spherically symmetric and in hydrostatic equilibrium $40+43$. The evolution is modeled with a modification to the equation of state ${ }^{44}$, where the $\mathrm{H}-\mathrm{He}$ EOS is based on $\mathrm{SCVH}^{45}$ with an extension to lower pressures and temperatures, and the heavy-element $\left(\mathrm{H}_{2} \mathrm{O} / \mathrm{SiO}_{2}\right)$ EOS is QEOS ${ }^{46}$ 47. Conductive opacities are from Cassisi et al. $(2007)^{48}$, and the molecular opacity is from Freedman et al. $(2007)^{49}$.

The planetary evolution is governed by the energy transport in the interior, which can occur via radiation, conduction, or convection. We use the standard Ledoux criterion ${ }^{50}$ to determine whether a region with composition gradients is stable against convection, i.e., $\nabla_{T}<\nabla_{a d}+B$, where $\nabla_{T}=d \log T / d \log P$, with $\nabla_{\text {ad }}$ and $B$ being the adiabatic temperature and composition gradient, respectively. If the composition gradient is such that the mean molecular weight increases towards the planetary center, then $B>0$ and the composition gradient could inhibit convection. For a homogeneous planet, $B=0$ and the Ledoux criterion reduces to the Schwarzschild criterion $\nabla_{T}<\nabla_{\text {ad }}$. A region that is Ledoux stable but Schwarzschild unstable could develop semi-convection. In that case, double-diffusive processes can lead to additional mixing 51 .

In the planet evolution code, convective mixing is treated via the mixing length theory (MLT), which provides a recipe to calculate $\nabla_{T}$ and the diffusion coefficient, fully determining the convective flux. The MLT requires the knowledge of a mixing length $l_{\mathrm{m}}=\alpha_{\mathrm{mlt}} H_{P}$, where $H_{P}$ is the pressure scale-height and $\alpha_{\text {mlt }}$ is a dimensionless parameter. The expected value of $\alpha_{\text {mlt }}$ for planets is poorly constrained. Following previous work on Jupiter's evolution with convective mixing we use $\alpha_{\text {mlt }}=0.1$ as our baseline. It is found that the mixing is relatively insensitive to the choice of the mixing length within about an order of magnitude. This is because its value does not directly determine when mixing occurs, but the mixing efficiency. To investigate the sensitivity of the results on this parameter we also included a model with $\alpha_{\text {mlt }}=10^{-3}$. While our conclusions on the diluted core are robust, a detailed and rigorous investigation on mixing in giant planets is clearly desirable, and will be presented in future work ${ }^{44}$.

The case of semi-convection is treated as a diffusive processes ${ }^{53}$ which requires the calculation of the temperature gradient and diffusion coefficient in the semi-convective region. The recipe includes a free parameter that can be interpreted as the layer-height of the double-diffusive region 5455 , which is unknown and could range over a few orders of magnitude. In the case where 
we include semi-convection, we set the value to $10^{-5}$ pressure scale heights, which is an intermediate value in the range given in the literature ${ }^{56}$.

The hydro-simulation of the giant impact sets the post-impact composition profile to be used by the evolution model. The initial temperature profile is crucial for determining the energy transport for the subsequent evolution. Since proto-Jupiter's thermal state at the time of impact is unknown, we consider various initial temperature profiles and explore how the mixing is affected by this choice. Giant planet formation calculations estimate the central temperature of proto-Jupiter to be $\sim 10^{4} \mathrm{~K}^{27}$. The exact temperature, however, is unknown and can change by a factor of a few. For determining the convective mixing efficiency such factors can lead to large differences in the long-term evolution and the final internal structure. Also, recent work has shown that accounting for the accretion shock during the runaway gas accretion phase can lead to a radiative envelope and a non-monotonic temperature profile in the deep interior ${ }^{26,27}$. We include this possibility in one of our models (H-radenv). Our nominal models use $\alpha_{\mathrm{mlt}}=0.1$, no semi-convection with the heavy elements being represented by water. A summary of the model parameters is given in Extended Data Table 2.

In Extended Data Figure 6 we present the starting models that are evolved to Jupiter's age. The solid and dashed lines correspond to the head-on and oblique (at an angle of 45 degrees) collisions, respectively. The temperatures are increasing towards the interior for all models except H-radenv, as explained above. Here, a temperature inversion occurs in the deep interior, corresponding to the location of the accretion shock during early runaway gas accretion. Note that in this model the location of the temperature-inversion occurs within the same region of the composition gradient, which supports the stability of the region against convection. While the exact location of the temperature jump is not well determined, it can be estimated due to the requirement of reaching the so called cross-over mass to enter the runaway phase $\mathrm{s}^{57}$. As the heavy-element fraction increases, the interior becomes hotter due to the change in opacity and the increase in density. If the collision is head-on, the composition gradient is shallower and extends farther into the envelope.

Extended Data Figures $7 \& 8$ show the density profiles after 4.56 Gyrs of evolution for the head-on and oblique collision, respectively. The crucial influence of the initial thermal profile on the mixing is clear: For the $\log T_{\mathrm{c}}[\mathrm{K}]=4.7$ head-on collision case $(\mathrm{H}-4.7)$, the end-result is a fully 
homogeneous Jupiter without a core. For the oblique impact, even the very steep composition gradient, with the highest temperatures, is insufficient to inhibit substantial mixing of the deep interior. The intermediate temperature profiles lead to varying degrees of mixing. In general, the head-on collision results in an extended core that is highly enriched in $\mathrm{H}-\mathrm{He}$, while for the oblique impact the core is more compact and less diluted. Despite a substantial fraction of proto-Jupiter being very hot in the model H-radenv, there is not enough mixing to erase the composition gradient. In this case, the envelope is radiative at early times when mixing would be most efficient. If a lower mixing length is chosen $(\mathrm{H}-4.5-\operatorname{low} \alpha)$, the composition gradient is less eroded and extends farther into the envelope. Because the energy transport is also affected by the chosen mixing length, Jupiter's interior is hotter and denser compared to H-4.5.

In H-4.5-semiconv, we consider the same model as $\mathrm{H}-4.5$ but allow semi-convective mixing. with a layer height of $10^{-5}$ pressure scale-heights. In this case, semi-convection is insufficient to overcome the stabilizing composition gradient. While some additional mixing occurs, particularly at early times, there are no semi-convective regions towards the end of the evolution. In other words, the final interior structure is such that the radiative regions are Schwarzschild and Ledoux stable. This demonstrates that also when semi-convection is included we infer a Jupiter with a diluted core.

In order to completely erase the composition gradient created by the giant impact the impact must be head-on with a very hot interior $(\sim 50,000 \mathrm{~K})$ with the heavy elements represented by water $(\mathrm{H}-4.7)$. In all the other models we consider, the stabilizing effect of the post-impact heavy-element distribution is inhibiting the development of convective instabilities resulting in an inhomogeneous Jupiter. Therefore, the typical outcome of the calculation is an interior structure that is not fully mixed and is characterized by several radiative-convective interfaces. Interestingly, the development of these interfaces seems to be a common occurrence when modelling Jupiter's evolution with composition gradients ${ }^{44 / 52}$. If the core is defined as the region that is heavy-element rich in comparison to the envelope, then most of our models imply that Jupiter has a diluted and extended core extending to $\sim 30 \%-50 \%$ of the planet's radius. All the oblique collisions lead to a relatively compact core since the initial composition gradient is very steep.

Figure 2 shows the models that best match the diluted-core density profile from Wahl et al. (2017) $)^{3}$ (H-4.5-rock, H-4.5, H-radenv). We find that for the head-on collision, a post-impact 
central temperature of $\sim 30,000 \mathrm{~K}$ leads to a current-state Jupiter with a diluted core $(\mathrm{H}-4.5$ and $\mathrm{H}-4.5$-rock). If the heavy elements are represented by rock $\left(\mathrm{SiO}_{2}\right)$, the diluted core extends farther into the envelope and is thus more consistent with a Jupiter structure with a diluted core. Another pathway to the diluted core is when Jupiter's deep interior is radiative due to the accretion shock as predicted by recent giant planet formation models ${ }^{27}$ (H-radenv). Videos that demonstrate the planetary evolution for three selected cases can be found in the Supplementary Information.

Data availability. The datasets generated and analysed during the current study are available from the corresponding authors upon reasonable request.

Code availability. The FLASH code is publicly available for download at http://flash.uchicago.edu/site/flash The implementation of giant impact simulations in the framework of FLASH is available upon request. The REBOUND code is publicly available for download at https://github.com/hannorein/rebound. The MESA code is an open source stellar evolution code and is publicly available at http://mesa.sourceforge.net. The modified version of the MESA code is not yet ready for public release - it will be presented in future work ${ }^{44}$. Gnuplot, Jupyter Notebook, Mathematica, VisIt and yt python package have been used for data reduction and presentation in this study. 


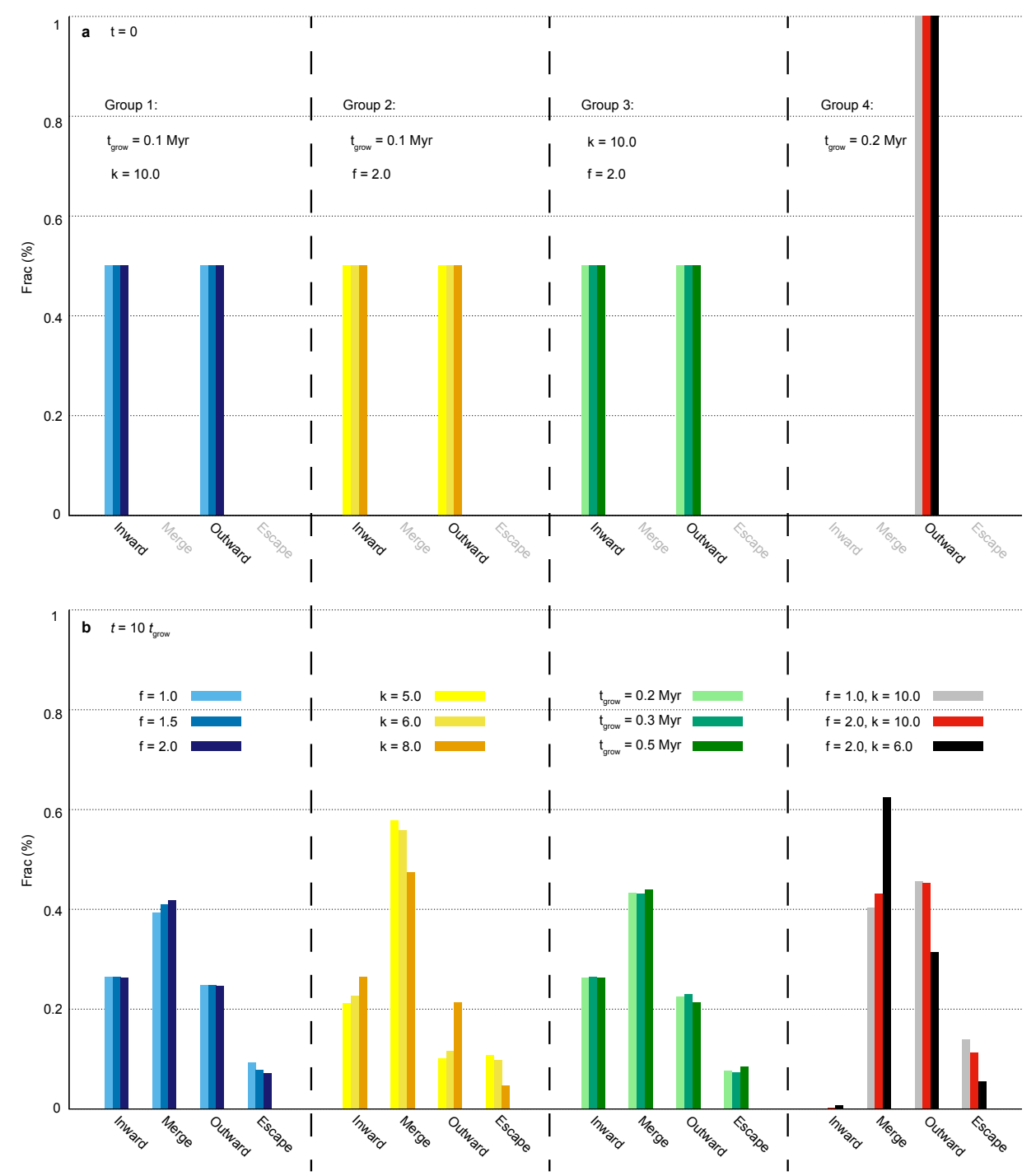

Figure 4: Extended Data Figure 1 - Statistics of outcomes of four planetary embryos under the influence of an emerging Jupiter. a, the initial configurations of four planetary embryos divided into four groups based on fixed parameters shown under the group numbers. In group 1-3, half of the embryos are placed inside Jupiter's orbit (labeled as "Inward"), the other half are outside Jupiter's orbit (labeled as "Outward"). In group 4, all embryos are outside Jupiter's orbit. The exact location of every embryo is shown in Table 1 in the Supplementary Information. bo the statistical outcomes of the dynamic evolution after $10 t_{\text {grow }}$. Because Jupiter's growth can substantially modify orbits of those embryos. Some embryos collided with Jupiter (labeled as "Merger"), and some have been ejected from the Solar system (labeled as "Escape"). Other embryos are labeled either "Inward" or "Outward" depending on their orbital locations inside or outside Jupiter's orbit. Colours indicate different choices of the free parameter displayed in legend in each group. 
a Group 1: $\quad t_{\text {grow }}=0.1 \mathrm{Myr}, \quad \mathrm{k}=10.0$
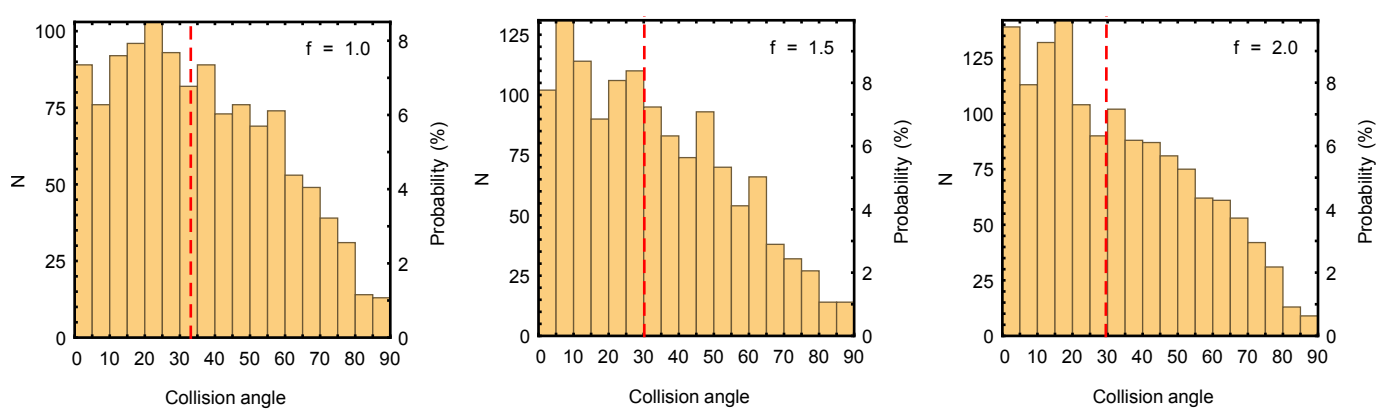

b Group 2: $t_{\text {grow }}=0.1 \mathrm{Myr}, \quad f=2.0$
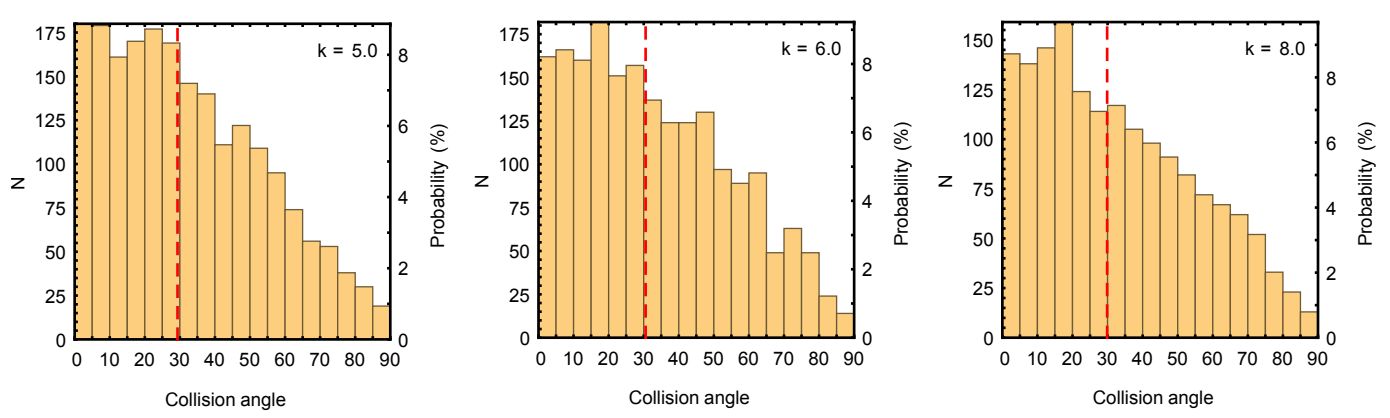

c Group 3: $k=10, f=2.0$
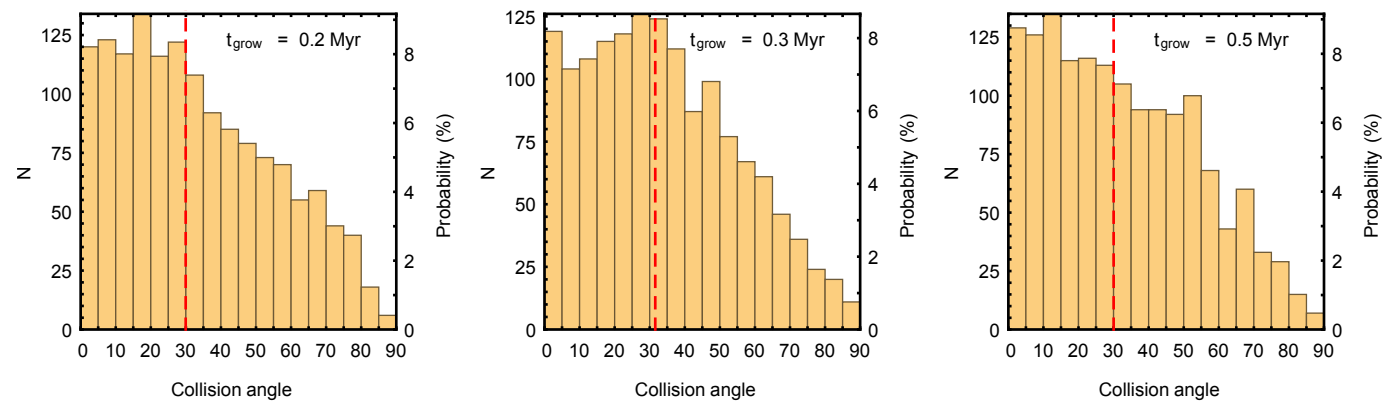

d Group 4: $\mathrm{t}_{\text {grow }}=0.2 \mathrm{Myr}$
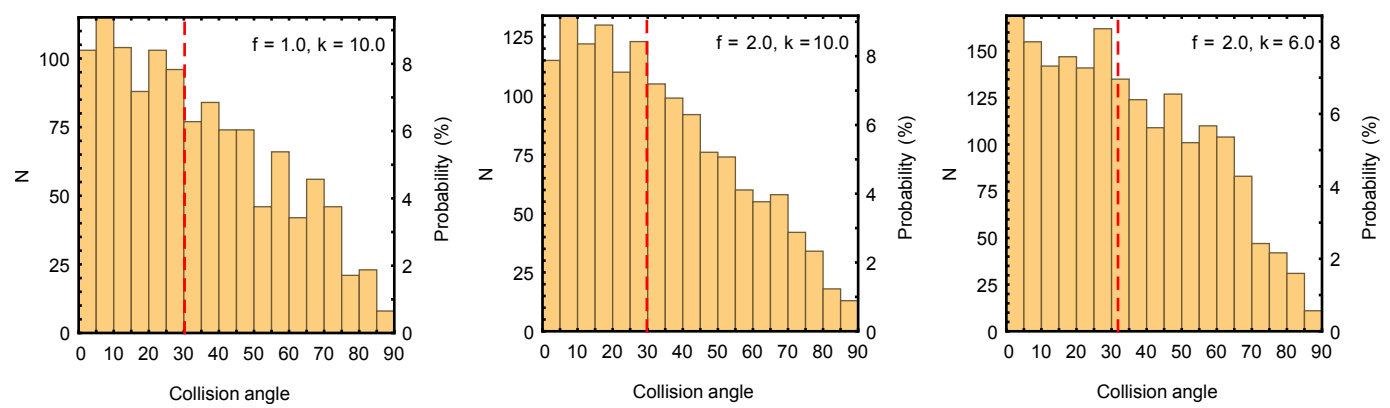

Figure 5: Extended Data Figure 2 - Histograms of collision angles of each data set presented in Extended Data Figure 1. a, group 1. b, group 2. c, group 3. d, group 4. The bin size is $5^{\circ}$, and there are 18 bins in each plot. The red dashed line indicate the median value in each case. The results suggest head-on collisions are more common than grazing ones. 


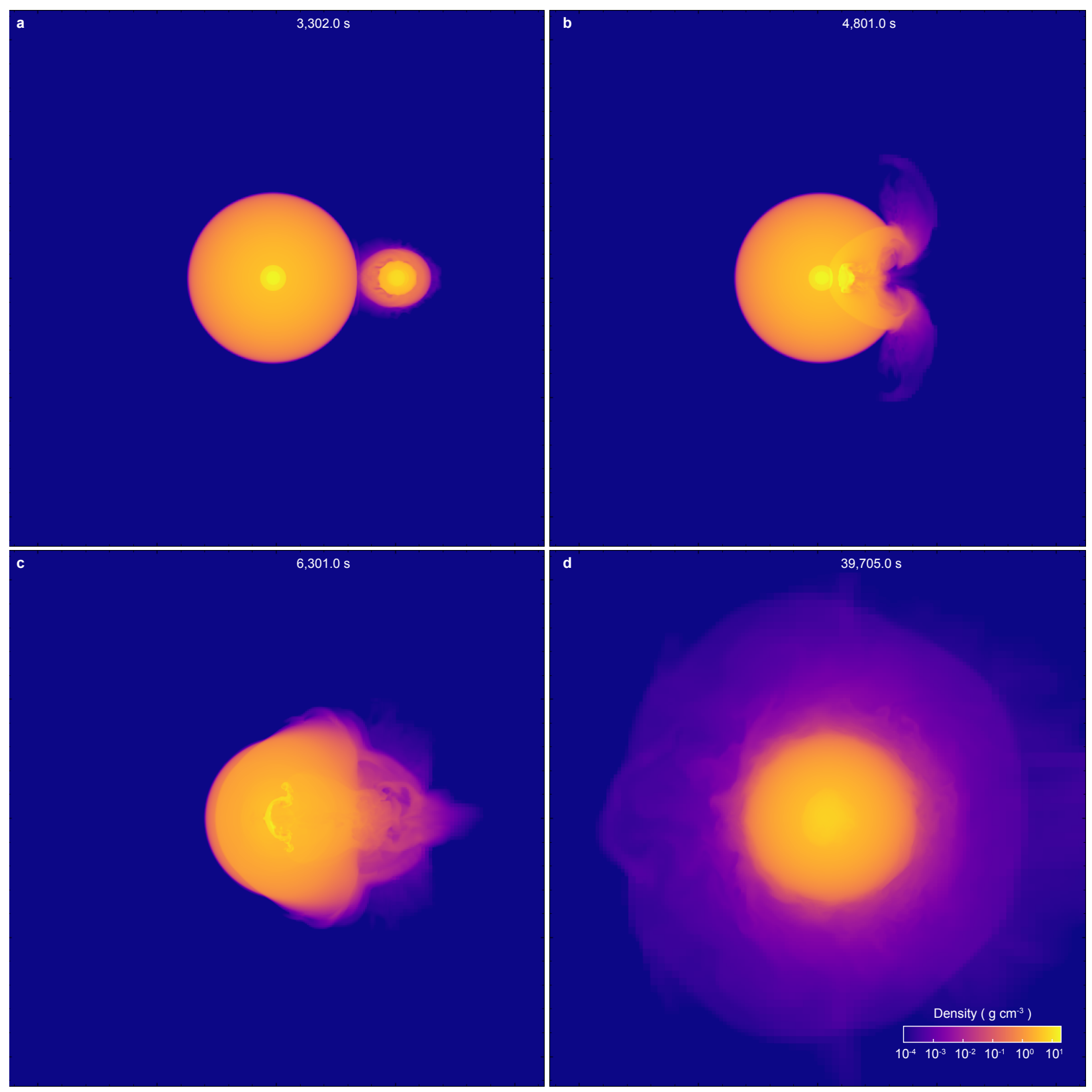

Figure 6: Extended Data Figure 3 - 2D snapshots of a merger between the proto-Jupiter with a $10 M_{\oplus}$ solid core and a $10 M_{\oplus}$ impactor. a, Density contours in the orbital plane before the impact; b, before the impactor arriving at the core; c, after the destruction of the core; d, at $\sim 10$ hours after the impact. 

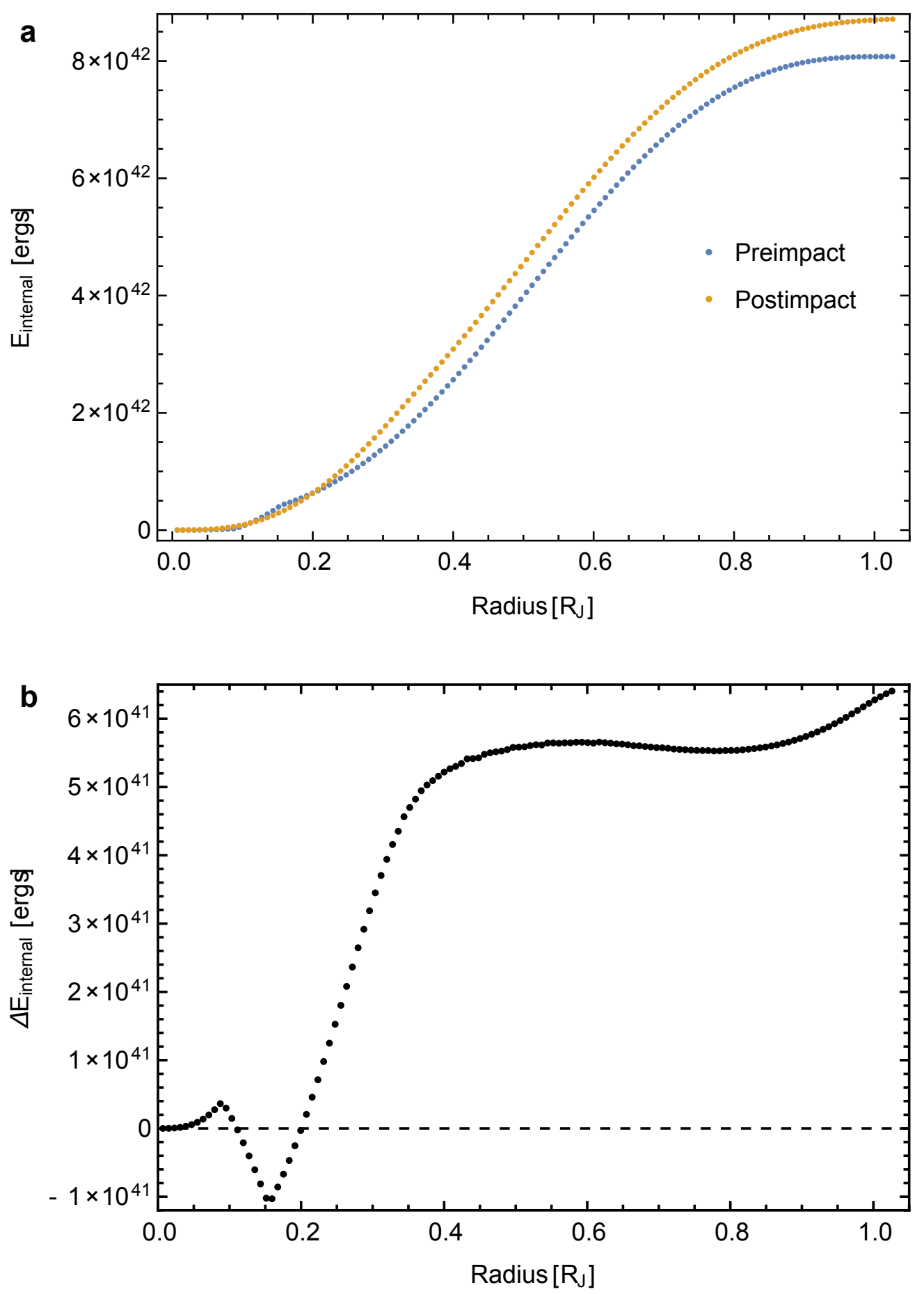

Figure 7: Extended Data Figure 4 - The change of internal energy caused by the merger in case-1. a, The enclosed internal energy of Jupiter before and after the impact as a function of radius. $\mathbf{b}$, The net change of enclosed internal energy of Jupiter as a function of radius. 


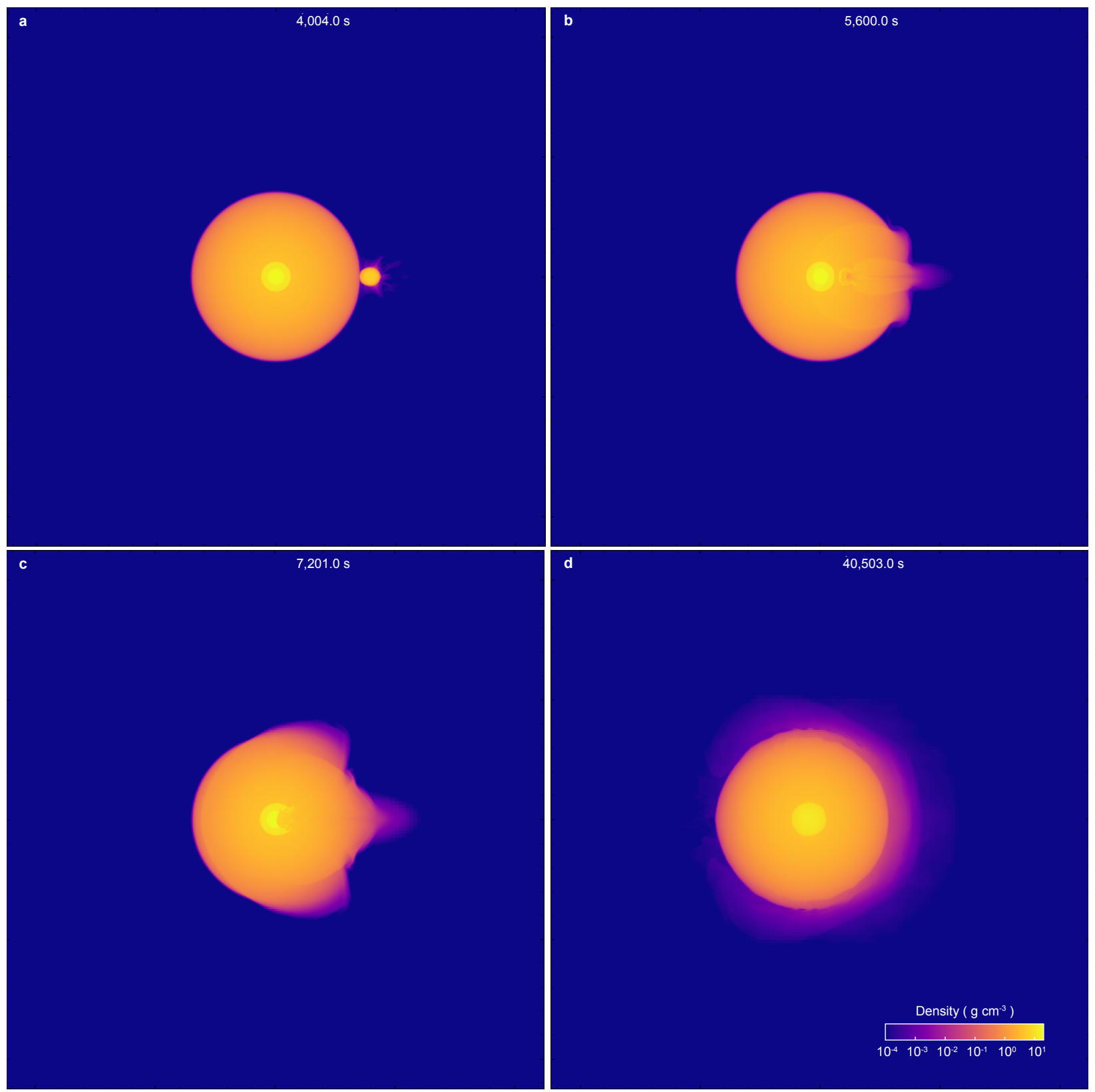

Figure 8: Extended Data Figure 5 - 2D snapshots of a merger between the primordial Jupiter with a $17 M_{\oplus}$ core and a $1 M_{\oplus}$ impactor. a, Density contours in the orbital plane before the impact; $\mathbf{b}$, before the impactor arriving at the core; $\mathbf{c}$, after the merger with the core; $\mathbf{d}$, at $\sim 10$ hours after the impact. 

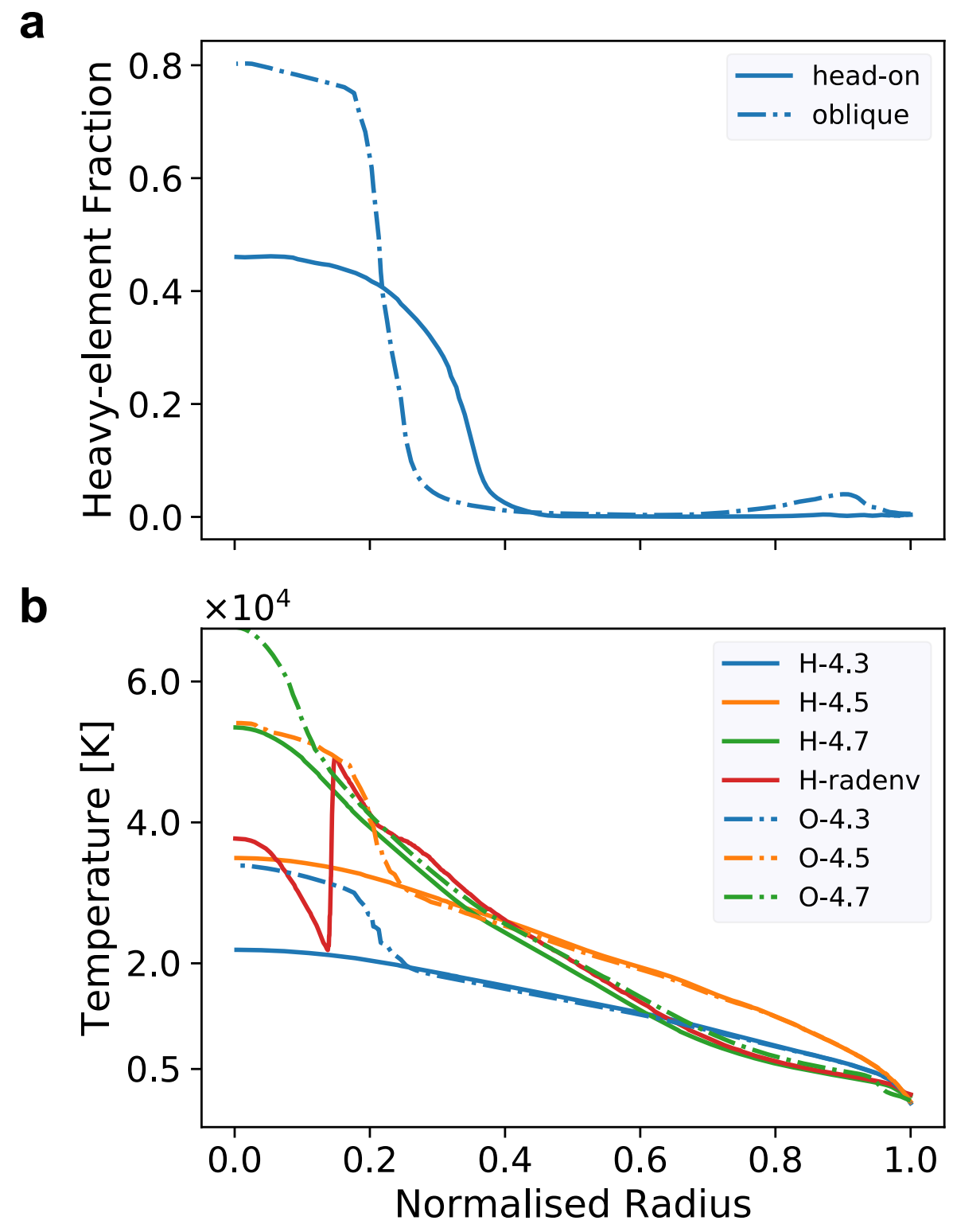

Figure 9: Extended Data Figure 6 - Initial conditions for post-impact evolution. a, the initial post-impact heavy-element profile and $\mathbf{b}$, temperature profiles of the models that are used for the thermal evolution. The heavy-element distribution is taken from the hydro simulation ten hours after the giant impact. Solid lines correspond to a head-on collision, while dashed-dotted lines show the result of an oblique collision at a 45 degree angle. The colours depict models with different initial thermal states. See text and Extended Data Table 2 for further details. 


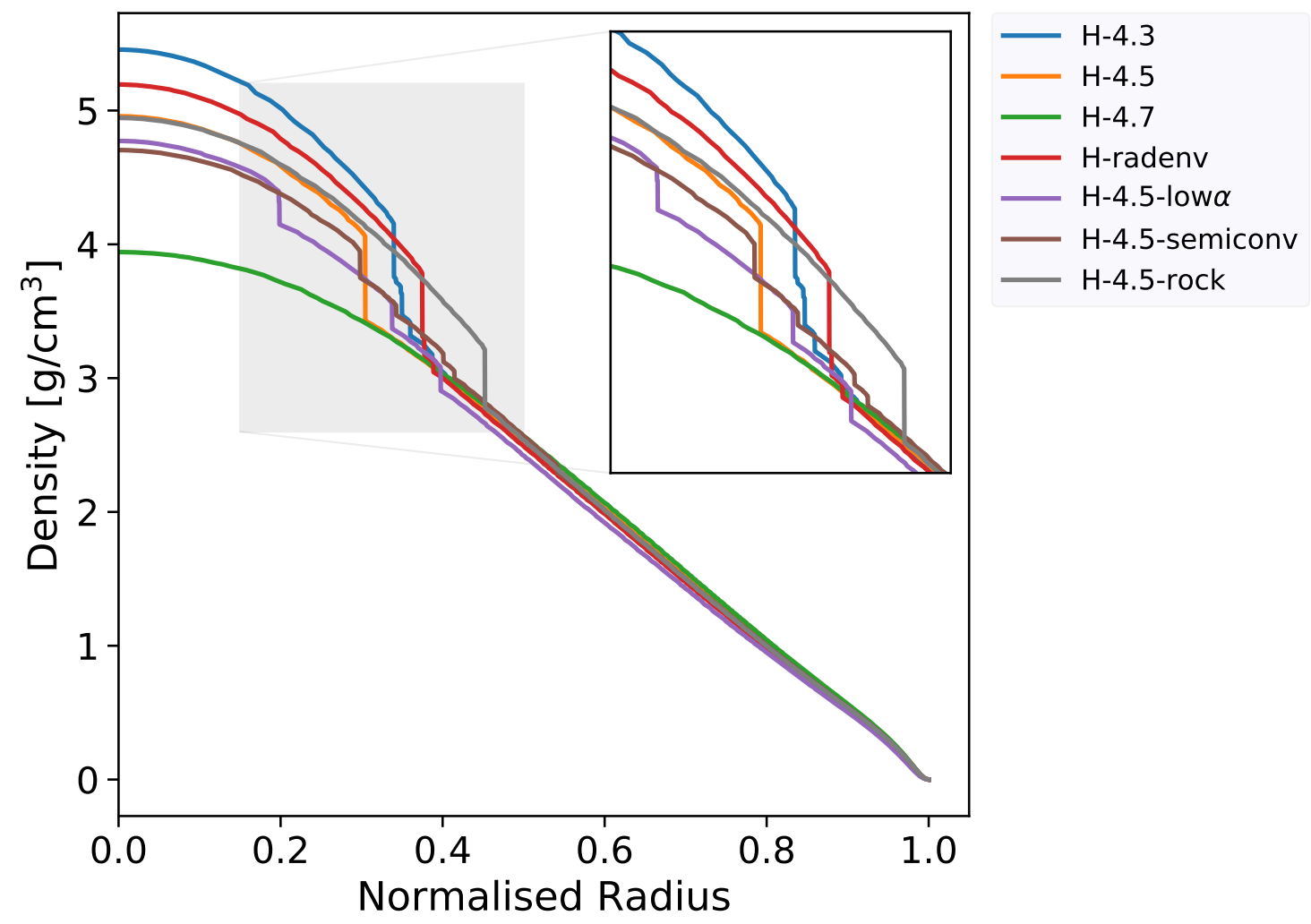

Figure 10: Extended Data Figure 7 - Density vs. normalized radius for the head-on collision after 4.56 Gyrs of evolution. The colors correspond to distinct model assumptions: H-4.3, H4.5, $\mathrm{H}-4.7$ correspond to initial thermal profiles with different central temperatures at the time of the impact, while $\mathrm{H}$-radenv assumes a proto-Jupiter with a radiative envelope. $\mathrm{H}-4.5$-low $\alpha$ uses a shorter mixing length, H-4.5-semiconv allows for semi-convective mixing, and in H-4.5-rock the heavy elements are represented by rock instead of water for EOS purposes. See text and Extended Data Table 2 for further details. 


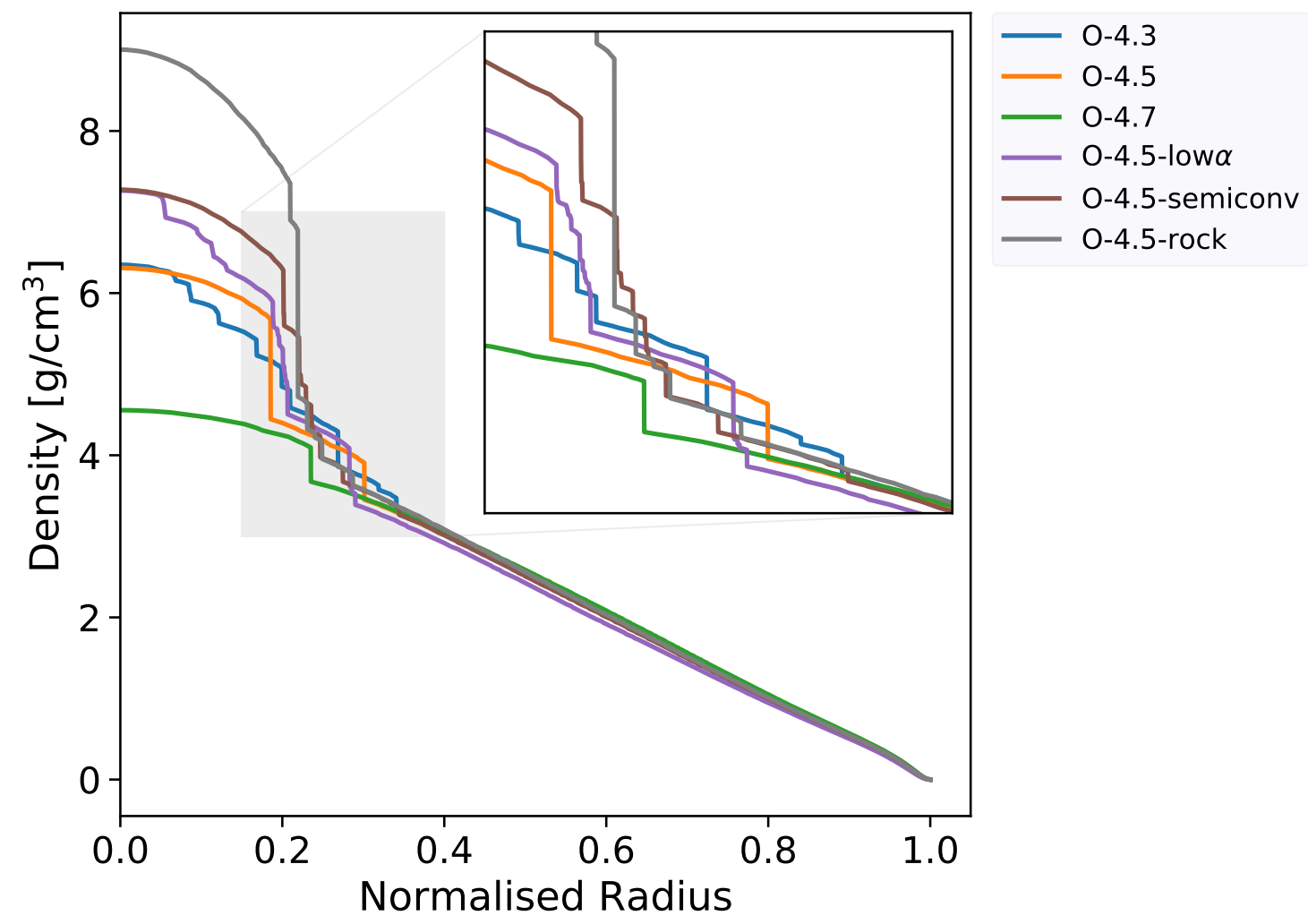

Figure 11: Extended Data Figure 8 - Density vs. normalized radius for the oblique collision after 4.56 Gyrs of evolution. The colors correspond to distinct model assumptions: O-4.3, O4.5, O-4.7 correspond to initial thermal profiles with different central temperatures at the time of the impact, while O-radenv assumes a proto-Jupiter with a radiative envelope. O-4.5-low $\alpha$ uses a shorter mixing length, O-4.5-semiconv allows for semi-convective mixing, and in O-4.5-rock the heavy elements are represented by rock instead of water for EOS purposes. See text and Extended Data Table 2 for further details. 


\begin{tabular}{|c|c|c|}
\hline$k$ & $q$ & $a[\mathrm{AU}]$ \\
\hline 5.0 & 1.11 & $4.19,4.66, \mathbf{5 . 2 0}, 5.79,6.45$ \\
\hline 6.0 & 1.14 & $4.01,4.56, \mathbf{5 . 2 0}, 5.92,6.74$ \\
\hline 8.0 & 1.19 & $3.68,4.37, \mathbf{5 . 2 0}, 6.18,7.35$ \\
\hline 10.0 & 1.24 & $3.37,4.19, \mathbf{5 . 2 0}, 6.46,8.02$ \\
\hline 6.0 & 1.14 & $\mathbf{5 . 2 0}, 5.92,6.74,7.67,8.73$ \\
\hline 10.0 & 1.24 & $\mathbf{5 . 2 0}, 6.46,8.02,9.95,12.36$ \\
\hline
\end{tabular}

Figure 12: Extended Data Table 1 - List of initial orbital semi-major axis of each embryo of our $N$-body simulation suite. The location of the embryo that grows into a Jupiter in each case is in bold face.

\begin{tabular}{|c|c|c|c|c|c|}
\hline Name & $\begin{array}{c}\log T_{\mathrm{c}} \\
{[\mathrm{K}]}\end{array}$ & $\begin{array}{c}\text { Collision } \\
\text { Type }\end{array}$ & $\begin{array}{c}\text { Heavy- } \\
\text { element type }\end{array}$ & $\alpha_{\mathrm{mlt}}$ & $\begin{array}{c}\text { Semi- } \\
\text { convection }\end{array}$ \\
\hline $\mathrm{H}-4.3$ & 4.3 & head-on & water & $10^{-1}$ & no \\
\hline $\mathrm{H}-4.5$ & 4.5 & head-on & water & $10^{-1}$ & no \\
\hline $\mathrm{H}-4.7$ & 4.7 & head-on & water & $10^{-1}$ & no \\
\hline $\mathrm{H}-$-radenv & - & head-on & water & $10^{-1}$ & no \\
\hline $\mathrm{H}-4.5-$-low $\alpha$ & 4.5 & head-on & water & $10^{-3}$ & no \\
\hline $\mathrm{H}-4.5$-semiconv & 4.5 & head-on & water & $10^{-1}$ & yes \\
\hline $\mathrm{H}-4.5-$-rock & 4.5 & head-on & rock & $10^{-1}$ & no \\
\hline O-4.3 & 4.3 & oblique & water & $10^{-1}$ & no \\
\hline O-4.5 & 4.5 & oblique & water & $10^{-1}$ & no \\
\hline O-4.7 & 4.7 & oblique & water & $10^{-1}$ & no \\
\hline O-4.5-low $\alpha$ & 4.5 & oblique & water & $10^{-3}$ & no \\
\hline O-4.5-semiconv & 4.5 & oblique & water & $10^{-1}$ & yes \\
\hline O-4.5-rock & 4.5 & oblique & rock & $10^{-1}$ & no \\
\hline
\end{tabular}

Figure 13: Extended Data Table 2 - Description of the evolutionary models that are discussed throughout this work. Note that the models H/O-radenv are unique in which they are the result of formation models of Jupiter ${ }^{26}$ that account for the accretion shock during the runaway gas accretion. 
1. Bolton, S. J. et al. The Juno Mission. Space Sci. Rev. 213, 5-37 (2017).

2. Folkner, W. M. et al. Jupiter gravity field estimated from the first two Juno orbits. Geophys. Res. Lett. 44, 4694-4700 (2017).

3. Wahl, S. M. et al. Comparing Jupiter interior structure models to Juno gravity measurements and the role of a dilute core. Geophys. Res. Lett. 44, 4649-4659 (2017).

4. Debras, F. \& Chabrier, G. New Models of Jupiter in the Context of Juno and Galileo. Astrophys. J. 872, 100 (2019).

5. Pollack, J. B. et al. Formation of the Giant Planets by Concurrent Accretion of Solids and Gas. Icarus 124, 62-85 (1996).

6. Ikoma, M., Nakazawa, K. \& Emori, H. Formation of Giant Planets: Dependences on Core Accretion Rate and Grain Opacity. Astrophys. J. 537, 1013-1025 (2000).

7. Helled, R. et al. Giant Planet Formation, Evolution, and Internal Structure. In Beuther, H., Klessen, R. S., Dullemond, C. P. \& Henning, T. (eds.) Protostars and Planets VI, 643 (2014).

8. Paardekooper, S.-J. \& Mellema, G. Planets opening dust gaps in gas disks. Astron. Astrophys. 425, L9-L12 (2004).

9. Levison, H. F., Thommes, E. \& Duncan, M. J. Modeling the Formation of Giant Planet Cores. I. Evaluating Key Processes. Astron. J. 139, 1297-1314 (2010).

10. Bitsch, B. et al. Pebble-isolation mass: Scaling law and implications for the formation of super-Earths and gas giants. Astron. Astrophys. 612, A30 (2018).

11. Guillot, T., Stevenson, D. J., Hubbard, W. B. \& Saumon, D. The interior of Jupiter (In: Jupiter. The planet, 2004).

12. Wilson, H. F. \& Militzer, B. Solubility of Water Ice in Metallic Hydrogen: Consequences for Core Erosion in Gas Giant Planets. Astrophys. J. 745, 54 (2012).

13. Stevenson, D. J. Structure of the Giant Planets: Evidence for Nucleated Instabilities and PostFormational Accretion. In Lunar and Planetary Science Conference, vol. 13 of Lunar and Planetary Science Conference, 770-771 (1982). 
14. Hori, Y. \& Ikoma, M. Gas giant formation with small cores triggered by envelope pollution by icy planetesimals. Mon. Not. R. Astron. Soc. 416, 1419-1429 (2011).

15. Lozovsky, M., Helled, R., Rosenberg, E. D. \& Bodenheimer, P. Jupiter's Formation and Its Primordial Internal Structure. Astrophys. J. 836, 227 (2017).

16. Guillot, T. THE INTERIORS OF GIANT PLANETS: Models and Outstanding Questions. Annu. Rev. Earth Planet. Sci. 33, 493-530 (2005).

17. Nettelmann, N., Becker, A., Holst, B. \& Redmer, R. Jupiter Models with Improved Ab Initio Hydrogen Equation of State (H-REOS.2). Astrophys. J. 750, 52 (2012).

18. Helled, R. \& Guillot, T. Interior Models of Saturn: Including the Uncertainties in Shape and Rotation. Astrophys. J. 767, 113 (2013).

19. Li, S.-L., Agnor, C. \& Lin, D. N. C. Embryo Impacts and Gas Giant Mergers. I. Dichotomy of Jupiter and Saturn's Core Mass. Astrophys. J. 720, 1161-1173 (2010).

20. Liu, S.-F., Agnor, C. B., Lin, D. N. C. \& Li, S.-L. Embryo impacts and gas giant mergers - II. Diversity of hot Jupiters' internal structure. Mon. Not. R. Astron. Soc. 446, 1685-1702 (2015).

21. Kokubo, E. \& Ida, S. Oligarchic Growth of Protoplanets. Icarus 131, 171-178 (1998).

22. Ida, S. \& Lin, D. N. C. Toward a Deterministic Model of Planetary Formation. I. A Desert in the Mass and Semimajor Axis Distributions of Extrasolar Planets. Astrophys. J. 604, 388-413 (2004).

23. Zhou, J.-L. \& Lin, D. N. C. Planetesimal Accretion onto Growing Proto-Gas Giant Planets. Astrophys. J. 666, 447-465 (2007).

24. Ida, S., Lin, D. N. C. \& Nagasawa, M. Toward a Deterministic Model of Planetary Formation. VII. Eccentricity Distribution of Gas Giants. Astrophys. J. 775, 42 (2013).

25. Fryxell, B. et al. FLASH: An Adaptive Mesh Hydrodynamics Code for Modeling Astrophysical Thermonuclear Flashes. Astrophys. J. Suppl. 131, 273-334 (2000).

26. Berardo, D. \& Cumming, A. Hot-start Giant Planets Form with Radiative Interiors. Astrophys. J. 846, L17 (2017). 
27. Cumming, A., Helled, R. \& Venturini, J. The primordial entropy of Jupiter. Mon. Not. R. Astron. Soc. 477, 4817-4823 (2018).

28. Helled, R. \& Stevenson, D. The Fuzziness of Giant Planets' Cores. Astrophys. J. Lett. 840, L4 (2017).

29. Thorngren, D. P. \& Fortney, J. J. Bayesian Analysis of Hot-Jupiter Radius Anomalies: Evidence for Ohmic Dissipation? Astron. J. 155, 214 (2018).

30. Rein, H. \& Liu, S.-F. REBOUND: an open-source multi-purpose N-body code for collisional dynamics. Astron. Astrophys. 537, A128 (2012).

31. Rein, H. \& Tamayo, D. WHFAST: a fast and unbiased implementation of a symplectic Wisdom-Holman integrator for long-term gravitational simulations. Mon. Not. R. Astron. Soc. 452, 376-388 (2015).

32. Rein, H. \& Spiegel, D. S. IAS15: a fast, adaptive, high-order integrator for gravitational dynamics, accurate to machine precision over a billion orbits. Mon. Not. R. Astron. Soc. 446, 1424-1437 (2015).

33. Asphaug, E., Agnor, C. B. \& Williams, Q. Hit-and-run planetary collisions. Nature 439, 155-160 (2006).

34. Lin, D. N. C. \& Ida, S. On the Origin of Massive Eccentric Planets. Astrophys. J. 477, 781-791 (1997).

35. Izidoro, A., Raymond, S. N., Morbidelli, A., Hersant, F. \& Pierens, A. Gas Giant Planets as Dynamical Barriers to Inward-Migrating Super-Earths. Astrophys. J. Lett. 800, L22 (2015).

36. Izidoro, A., Morbidelli, A., Raymond, S. N., Hersant, F. \& Pierens, A. Accretion of Uranus and Neptune from inward-migrating planetary embryos blocked by Jupiter and Saturn. Astron. Astrophys. 582, A99 (2015).

37. Liu, S.-F., Hori, Y., Lin, D. N. C. \& Asphaug, E. Giant Impact: An Efficient Mechanism for the Devolatilization of Super-Earths. Astrophys. J. 812, 164 (2015).

38. Melosh, H. J. Impact cratering: A geologic process (Research supported by NASA. New York, Oxford University Press (Oxford Monographs on Geology and Geophysics, No. 11), 1989, 253 p., 1989). 
39. Liu, S.-F., Guillochon, J., Lin, D. N. C. \& Ramirez-Ruiz, E. On the Survivability and Metamorphism of Tidally Disrupted Giant Planets: The Role of Dense Cores. Astrophys. J. 762, 37 (2013).

40. Paxton, B. et al. Modules for Experiments in Stellar Astrophysics (MESA). Astrophys. J. Suppl. 192, 1-110 (2011).

41. Paxton, B. et al. Modules for experiments in stellar astrophysics (MESA): Planets, oscillations, rotation, and massive stars. Astrophys. J. Suppl. 208 (2013).

42. Paxton, B. et al. Modules for Experiments in Stellar Astrophysics (MESA): Binaries, pulsations, and explosions. Astrophys. J. Suppl. 220, 1-43 (2015).

43. Paxton, B. et al. Modules for Experiments in Stellar Astrophysics (MESA): Convective Boundaries, Element Diffusion, and Massive Star Explosions. Astrophys. J. Suppl. 234, 34 (2018).

44. Müller, S., Cumming, A. \& Helled, R. A systematic study of convective mixing in gas giant planets (2019, in prep.).

45. Saumon, D., Chabrier, G. \& van Horn, H. M. An Equation of State for Low-Mass Stars and Giant Planets. Astrophys. J. Suppl. 99, 713 (1995).

46. More, R. M., Warren, K. H., Young, D. A. \& Zimmerman, G. B. A new quotidian equation of state (QEOS) for hot dense matter. Phys. Fluids 31, 3059-3078 (1988).

47. Vazan, A., Kovetz, A., Podolak, M. \& Helled, R. The effect of composition on the evolution of giant and intermediate-mass planets. Mon. Not. R. Astron. Soc. 434, 3283-3292 (2013).

48. Cassisi, S., Potekhin, A. Y., Pietrinferni, A., Catelan, M. \& Salaris, M. Updated ElectronConduction Opacities: The Impact on Low-Mass Stellar Models. Astrophys. J. 661, 10941104 (2007).

49. Freedman, R. S., Marley, M. S. \& Lodders, K. Line and Mean Opacities for Ultracool Dwarfs and Extrasolar Planets. Astrophys. J. Suppl. 174, 504-513 (2008).

50. Ledoux, W. P. \& P. Stellar Models with Convection and with Discontinuity of the Mean Molecular. Astrophys. J. 105, 305 (1947). 
51. Rosenblum, E., Garaud, P., Traxler, A. \& Stellmach, S. Turbulent Mixing and Layer Formation in Double-diffusive Convection: Three-dimensional Numerical Simulations and Theory. Astrophys. J. 731, 66 (2011).

52. Vazan, A., Helled, R. \& Guillot, T. Jupiter's evolution with primordial composition gradients. Astron. Astrophys. 610, L14 (2018).

53. Langer, N., Sugimoto, D. \& Fricke, K. J. Semiconvective diffusion and energy transport. Astron. Astrophys 126, 207-208 (1983).

54. Wood, T. S., Garaud, P. \& Stellmach, S. A new model for mixing by double-diffusive convection (semi-convection). II. the transport of heat and composition through layers. Astrophys. $J$. 768 (2013).

55. Radko, T. et al. Double-Diffusive Recipes. Part I: Large-Scale Dynamics of Thermohaline Staircases. J. Phys. Oceanogr. 44, 1269-1284 (2014).

56. Leconte, J. \& Chabrier, G. A new vision of giant planet interiors: Impact of double diffusive convection. Astron. Astrophys. 540, 20 (2012).

57. Baraffe, I., Chabrier, G., Fortney, J. J. \& Sotin, C. Planetary Internal Structures. Protostars and Planets VI 763-786 (2014). 